\title{
Giant spin-orbit-induced spin splitting in two-dimensional transition-metal dichalcogenide semiconductors
}

\author{
Z. Y. Zhu, Y. C. Cheng, and U. Schwingenschlögl* \\ Physical Sciences and Engineering Division, KAUST, Thuwal 23955-6900, Kingdom of Saudi Arabia
}

(Received 13 September 2011; published 14 October 2011)

\begin{abstract}
Fully relativistic first-principles calculations based on density functional theory are performed to study the spin-orbit-induced spin splitting in monolayer systems of the transition-metal dichalcogenides $\mathrm{MoS}_{2}, \mathrm{MoSe}_{2}$, $\mathrm{WS}_{2}$, and $\mathrm{WSe}_{2}$. All these systems are identified as direct-band-gap semiconductors. Giant spin splittings of 148-456 meV result from missing inversion symmetry. Full out-of-plane spin polarization is due to the two-dimensional nature of the electron motion and the potential gradient asymmetry. By suppression of the Dyakonov-Perel spin relaxation, spin lifetimes are expected to be very long. Because of the giant spin splittings, the studied materials have great potential in spintronics applications.
\end{abstract}

DOI: 10.1103/PhysRevB.84.153402

PACS number(s): 75.70.Tj, 73.22.-f

\section{INTRODUCTION}

When electrons move through an effective magnetic field induced by an electric field perpendicular to the direction of motion, lifting of spin degeneracy is expected. Spin splitting by spin-orbit interaction results from the asymmetrical potential gradient (the electric field) in the vicinity of atomic sites. ${ }^{1}$ Two alternative mechanisms exist for this spin-orbit spin splitting, related to different sources of the electric field asymmetry: (i) the host crystal field (Dresselhaus effect) ${ }^{2}$ and (ii) the interface or surface electric field (Rashba effect) ${ }^{3}$ Both cases can be utilized in spintronics devices. ${ }^{4-6}$ Application, however, is hampered by the modest spin-orbit interaction of the currently available materials, which necessitates low temperatures for device operation, long channel lengths, and ultrahigh purity materials to avoid spin-flip scattering. ${ }^{7}$ In this respect, materials with an enhanced spin-orbit-induced spin splitting are highly desirable.

The semiconducting transition-metal dichalcogenides $M X_{2}(M=\mathrm{Mo}, \mathrm{W}$ and $X=\mathrm{S}, \mathrm{Se})$ have attracted a great deal of interest because of distinctive electronic, optical, and catalytic properties as well as the importance for dry lubrication. ${ }^{8-14}$ The materials crystallize in a layered $2 H$ prototype structure with space group $P 6_{3} / m m c\left(D_{6 h}^{4}\right)$, which consists of weakly van der Waals-bonded $X-M-X$ slabs. ${ }^{15,16}$ In such a slab, an intermediate layer of hexagonally arranged $M$ atoms is sandwiched between two layers of $X$ atoms due to strong ionic-covalent bonds, giving rise to a trigonal prismatic arrangement. The quasi-two-dimensional nature of the $2 \mathrm{H}-\mathrm{MX_{2 }}$ compounds enables the creation of a stable $M X_{2}$ monolayer by micromechanical cleavage and liquid exfoliation. ${ }^{17,18}$ The trigonal prismatic coordination of the bulk is maintained, whereas the symmetry of an $M X_{2}$ monolayer is reduced to $P \overline{6} m 2\left(D_{3 h}^{1}\right)$ because of a loss of inversion symmetry. Recent experiments by optical spectroscopy have shown an indirect-direct-band-gap transition in $\mathrm{MoS}_{2}$, due to a missing interlayer interaction. ${ }^{12}$ A field-effect transistor based on a $\mathrm{MoS}_{2}$ monolayer is described in Ref. 13. An illustration of the $2 \mathrm{H}-\mathrm{MX}_{2}$ unit cell and Brillouin zone is given in Fig. 1.

We report fully relativistic calculations within density functional theory on the spin-orbit-induced spin splitting in monolayers of the four transition-metal dichalcogenides $\mathrm{MoS}_{2}, \mathrm{MoSe}_{2}, \mathrm{WS}_{2}$, and $\mathrm{WSe}_{2}$. For all these systems, we obtain a direct-band-gap semiconductor with the valence-band maximum (VBM) and conduction-band minimum (CBM) located at the $\mathrm{K}$ point. A sizable spin splitting due to the spin-orbit interaction is found, and its implications for the material properties are analyzed.

\section{METHODOLOGY}

We apply the full-potential linearized augmented-planewave approach of the WIEN $2 \mathrm{~K}$ code,${ }^{19}$ which has been used successfully in various instances for describing low-dimensional systems. $^{20,21}$ A periodic slab geometry with a 30 - $\AA$-thick vacuum layer is used. Both the in-plane lattice constant $a$ and the positional parameter $z$ are optimized up to a force convergence of $0.5 \mathrm{mRy} /$ Bohr. For comparison, the electronic band structures of the bulk compounds are calculated with the experimental lattice constants $a$ and $c$ and the optimized $z$ value. The same values of $R_{\mathrm{mt}}=2.1$ a.u., $R_{\mathrm{mt}} K_{\max }=$ 7 , and $\ell_{\max }=10$ and exchange-correlation functional ${ }^{22}$ are used in each calculation. Concerning the reciprocal space, $16 \times 16 \times 1$ and $16 \times 16 \times 3$ meshes are employed for the monolayer and bulk systems, respectively. The spin-orbit coupling is included in terms of the second-variational method with scalar-relativistic orbitals as a basis.

Structural deformation is expected to affect the $M X_{2}$ monolayer systems due to absence of the van der Waals interlayer interaction, ${ }^{17,23,24}$ although the latter usually is weak. The lattice parameters of the fully relaxed monolayer systems are shown in Table I and compared to the bulk materials. The optimized positional parameters of the latter are close to the experimental value of $z=0.129 .{ }^{25}$ Experimental data are not available for free monolayers. Our data point to an expansion of $a$ by about $1 \%$, whereas the separation $d$ of the $M$ and $X$ layers hardly changes, except for a $0.3 \%$ decrease for $\mathrm{WS}_{2}$.

\section{RESULTS AND DISCUSSION}

Electronic band structures are calculated for both the $M X_{2}$ monolayers, compare Figs. 2(a)-2(d), and the corresponding bulk materials (not shown here). The absence of interlayer interaction leads to strong modifications of the electronic and optical properties in all the monolayer systems. They are found to be direct-band-gap semiconductors with the VBM and CBM 


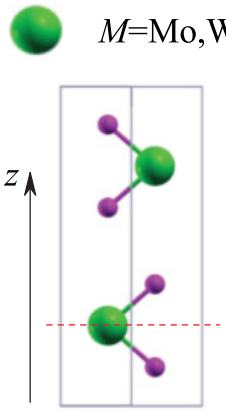

(a)

\section{$X=\mathrm{S}, \mathrm{Se}$}

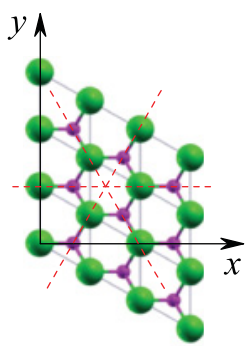

(b)

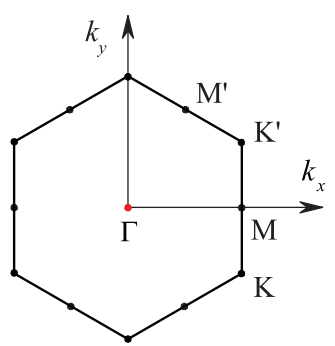

(c)
FIG. 1. (Color online) (a) Side view of the $2 H-M X_{2}(M=\mathrm{Mo}, \mathrm{W}$ and $X=\mathrm{S}, \mathrm{Se}$ ) unit cell, containing two $M X_{2}$ monolayers. (b) Top view. (c) First Brillouin zone of the $M X_{2}$ monolayer. Dashed lines indicate mirror planes.

at the $\mathrm{K}$ point of the Brillouin zone, in contrast to the indirect band gaps of the bulk materials with the VBM at the $\Gamma$ point and the CBM at about $0.55 \Gamma-\mathrm{K} \cdot{ }^{15,25-30}$ An indirect-directband-gap crossover with decreasing sample thickness has been exemplified for $\mathrm{MoS}_{2}$ experimentally. ${ }^{12}$ While the CBM of a $\mathrm{WS}_{2}$ monolayer has not been studied, a shift of the VBM from the $\Gamma$ to the $\mathrm{K}$ point due to the absence of interlayer interaction has been demonstrated by angle-resolved photoelectron spectroscopy. ${ }^{29}$ There is no experimental information available for the Se monolayers. However, because of similar crystal structures as well as chemical compositions and the good agreement between theory and experiment for $\mathrm{MoS}_{2}$ and $\mathrm{WS}_{2}$, we expect our results to be accurate also for these systems.

Turning to the spin-orbit interaction, Kramer's degeneracy $\left[E^{\uparrow}(\vec{k})=E^{\downarrow}(\vec{k})\right]$ is established in the $M X_{2}$ bulk by the combination of time-reversal $\left[E^{\uparrow}(\vec{k})=E^{\downarrow}(-\vec{k})\right]$ and inversion symmetry $\left[E^{\uparrow}(\vec{k})=E^{\uparrow}(-\vec{k})\right] .{ }^{31,32}$ However, the absence of inversion symmetry in an $M X_{2}$ monolayer system is expected to cause the energy bands to develop a spin splitting, except for $\vec{k}$ points with special symmetry in the Brillouin zone. As shown in Figs. 2(a)-2(d), the spin-orbit interaction breaks the spin degeneracy of the valence and conduction bands along the line $\Gamma-\mathrm{K}$. The materials exhibit a similar behavior but different magnitudes of the spin splitting. As it has the most prominent splitting, we will concentrate on $\mathrm{WSe}_{2}$ in the following. The quantum confinement in our quasi-two-

TABLE I. Structural parameters of bulk and monolayer $M X_{2}$ : inplane $(a)$ and out-of-plane $(c)$ lattice parameters, positional parameter $(z)$, and separation of the $M$ and $X$ layers $(d)$. For the monolayer system, both $a$ and $c$ have been optimized. For the bulk, we show the experimental values of $a$ and $c$ (Refs. 15,25) and the optimized values of $z$ and $d$. The experimental value is $z=0.129$ for all bulk compounds. $^{25}$

\begin{tabular}{lccccccc}
\hline \hline & \multicolumn{2}{c}{ Monolayer } & & \multicolumn{4}{c}{ Bulk } \\
\cline { 2 - 5 } & $a(\AA)$ & $d(\AA)$ & & $a(\AA)$ & $c(\AA)$ & $z$ & $d(\AA)$ \\
\hline $\mathrm{MoS}_{2}$ & 3.193 & 1.567 & & 3.160 & 12.295 & 0.127 & 1.568 \\
$\mathrm{MoSe}_{2}$ & 3.313 & 1.673 & & 3.288 & 12.900 & 0.130 & 1.672 \\
$\mathrm{WS}_{2}$ & 3.197 & 1.575 & & 3.153 & 12.323 & 0.128 & 1.580 \\
$\mathrm{WSe}_{2}$ & 3.310 & 1.685 & & 3.280 & 12.950 & 0.130 & 1.686 \\
\hline \hline
\end{tabular}

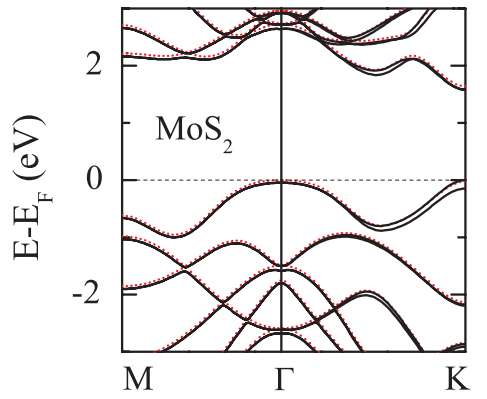

(a)

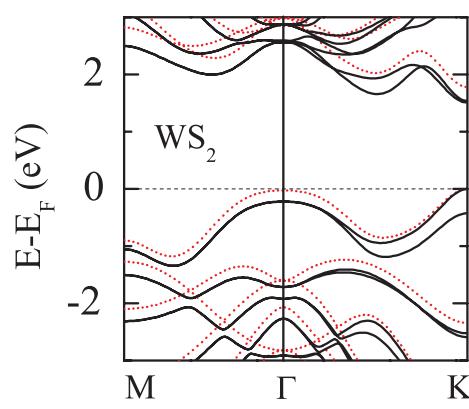

(c)

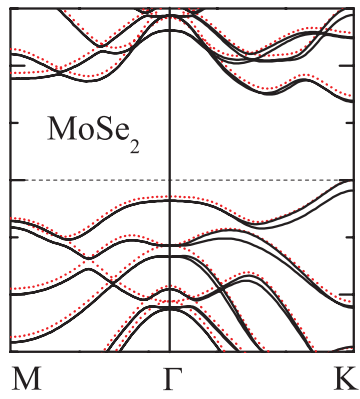

(b)

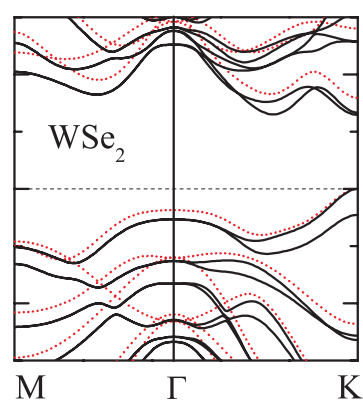

(d)
FIG. 2. (Color online) Electronic band structures calculated for the $M X_{2}$ monolayer systems with (solid line) and without (dotted line) inclusion of the spin-orbit interaction.

dimensional monolayers leads to very small dispersions ( $<1 \mathrm{meV}$ ) of the electronic bands along $k_{z}$. Therefore, all features of the band structure are covered by the $k_{z}=0$ plane.

Before continuing with the discussion of our numerical results, we briefly study the spin splitting by symmetry arguments. Spin degeneracy at the $\Gamma$ point results from the timereversal symmetry alone. ${ }^{32} \mathrm{~A}$ combination of time-reversal and translational symmetry results in zero splitting at the M point, whereas the spin splitting of a general $\vec{k}$ point is determined by time-reversal and $D_{3 h}$ point-group symmetry. The $D_{3 h}$ group combines the $C_{s}$, see Fig. 1(a), and $C_{3 v}$, see Fig. 1(b), symmetry groups. Considering the pure in-plane nature of the electron motion in our systems due to the quantum confinement, the $C_{3 v}$ symmetry alone leads to the spin splitting, ${ }^{33}$

$$
\Delta\left(k_{\|}, \theta\right)=\left[\alpha^{2}\left(k_{\|}\right)+\beta^{2}\left(k_{\|}\right) \sin ^{2} 3 \theta\right]^{1 / 2}
$$

with the spin polarization vector $\vec{P}_{ \pm}\left(k_{\|}, \theta\right)=$ $\Delta^{-1}\left(k_{\|}, \theta\right) \cdot\left[ \pm \alpha\left(k_{\|}\right) \sin \theta, \mp \alpha\left(k_{\|}\right) \cos \theta, \mp \beta\left(k_{\|}\right) \sin 3 \theta\right]$. Here, the out-of-plane and in-plane potential gradient asymmetries are represented by the coefficients $\alpha>0$ and $\beta>0$, respectively. The subscripts + and - denote spin-splitted states with higher and lower energy at the VBM. An additional $C_{s}$ symmetry dictates $\alpha\left(k_{\|}\right)=0$, which leads to the spin splitting $\Delta\left(k_{\|}, \theta\right)=\beta\left(k_{\|}\right)|\sin 3 \theta|$ and a full out-of-plane spin polarization $\vec{P}_{ \pm}\left(k_{\|}, \theta\right)=[0,0, \operatorname{sgn}(\mp \sin 3 \theta)]$.

The above symmetry arguments are partially reflected by our first-principles results. In Figs. 3(a) and 3(b), we address the magnitude of the spin splitting $\Delta$ in the $k_{z}=0$ plane of the $\mathrm{WSe}_{2}$ monolayer for the uppermost valence band (UVB) and the lowermost conduction band (LCB), respectively. A $C_{6}$ symmetry is clearly visible. Zero spin splitting is seen 


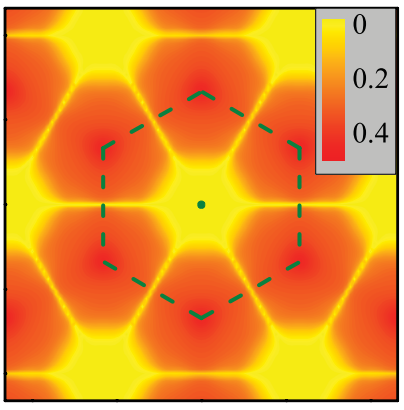

(a)

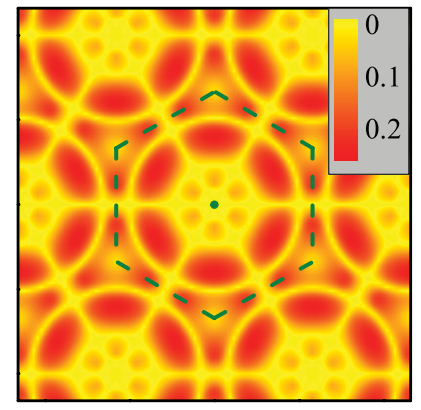

(b)

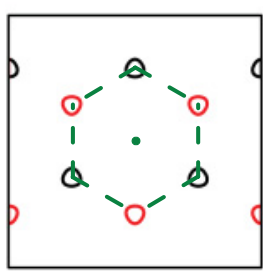

(c)

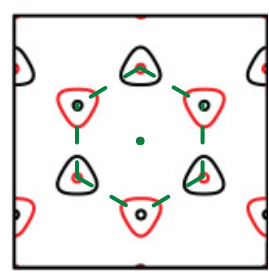

(d)

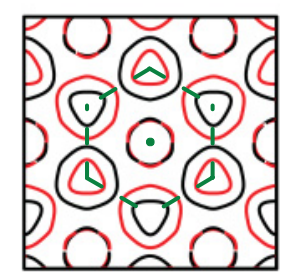

(e)
FIG. 3. (Color online) Spin splitting within the $k_{z}=0$ plane as obtained for (a) the uppermost valence band as well as (b) the lowermost conduction band of a $\mathrm{WSe}_{2}$ monolayer. Dashed lines highlight the first Brillouin zone. Energy isosurfaces are shown for binding energies of (c) 0.2 , (d) 0.5 , and (e) $0.8 \mathrm{eV}$. Spin orientations are indicated by different colors.

for $\theta=n \pi / 3, n \in \mathbb{N}_{0}$, which is consistent with the spin degeneracy of our band structure along the high-symmetry line $\Gamma$-M; see Fig. 2. The maximal spin splitting always appears for $\theta=(2 n+1) \pi / 6$ due to the $|\sin 3 \theta|$ dependence of $\Delta$. In Figs. 3(c)-3(e), we give energy isosurfaces for the binding energies $0.2,0.5$, and $0.8 \mathrm{eV}$. By the alternating spin orientations (indicated by different colors), the symmetry is reduced to $C_{3}$, which agrees with the $\sin 3 \theta$ dependence of the spin polarization. The full out-of-plane spin polarization is a consequence of the pure in-plane electron motion and the potential gradient asymmetry. As a result, unusually long spin lifetimes are expected for spins normal to the $M X_{2}$ monolayers, since the Dyakonov-Perel spin relaxation is suppressed. ${ }^{34}$

We show in Figs. 4(a) and 4(b) the $k_{\|}$dependence of the spin splitting $\Delta$ for the UVB and the LCB along the line $\Gamma-\mathrm{K}$, where the maximal splitting is found. Both bands exhibit a complicated behavior. For the UVB, $\Delta\left(k_{\|}\right)$first becomes slightly negative and then rises continuously up to its maximum (456 meV) at the K point. For the LCB, $\Delta\left(k_{\|}\right)$changes its sign several times, with a steep minimum about midway and a local minimum at the $\mathrm{K}$ point. These results are in line with the distributions shown in Figs. 3(a) and 3(b). We observe a similar behavior also for the other three compounds under investigation, but with different magnitudes of the spin splitting. The maximal spin splitting of the UVB at the K point amounts to $148,183,426$, and $456 \mathrm{meV}$ for the $\mathrm{MoS}_{2}, \mathrm{MoSe}_{2}, \mathrm{WS}_{2}$, and $\mathrm{WSe}_{2}$ monolayer, respectively. For $X=$ Se the splitting is slightly larger than for $X=\mathrm{S}$, while for $M=\mathrm{W}$ it is much larger than for $M=$ Mo. This observation originates from the fact that the spin-orbit coupling is stronger for heavier atoms. The magnitude of the spin splitting $\Delta$ in our $M X_{2}$ monolayer

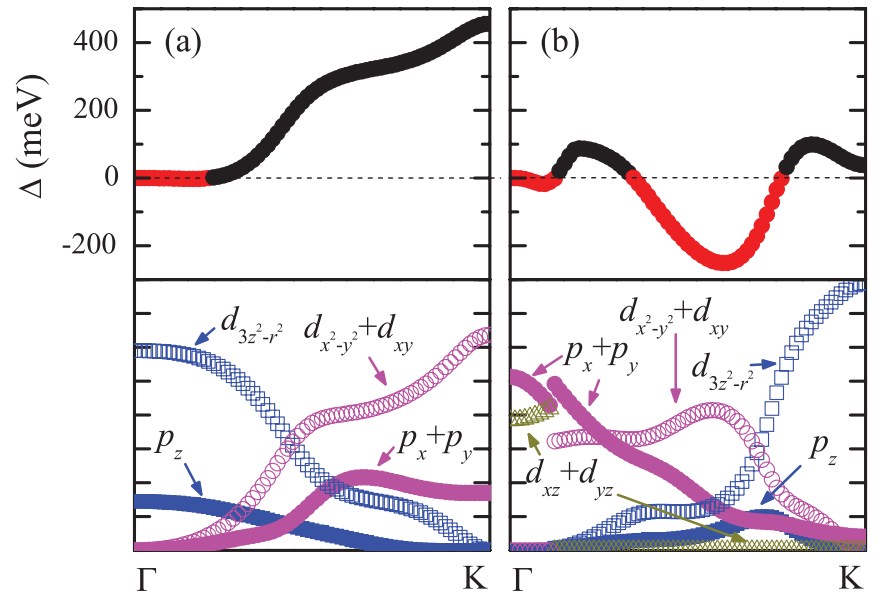

FIG. 4. (Color online) Spin splitting as a function of $k_{\|}$along the line $\Gamma-\mathrm{K}$ : (a) uppermost valence band as well as (b) lowermost conduction band. The sign reflects the spin orientation. Orbitally resolved contributions of $\mathrm{Se}$ and $\mathrm{W}$ are presented in the bottom panels (in arbitrary units).

systems is much larger than typical values for the Rashba spin splitting in the two-dimensional electron gas of conventional III-V and II-VI semiconductor quantum wells $(<30 \mathrm{meV}){ }^{35,36}$ Instead, it is fully comparable to the enhanced surface Rashba splitting (of some $100 \mathrm{meV}$ ) in $\mathrm{Au}(111),{ }^{31} \mathrm{Bi}(111),{ }^{32}$ $\mathrm{Pb} / \mathrm{Ge}(111),{ }^{37} \mathrm{Bi}_{2} \mathrm{Se}_{3}(001),{ }^{38}$ and W(110) (Ref. 39) surfaces.

An explanation of the observed $k_{\|}$dependence of $\Delta$ is provided by an investigation of the orbital contributions to the $\mathrm{UVB}$ and LCB. For the $\mathrm{WSe}_{2}$ monolayer system, results on the Se and $\mathrm{W}$ contributions are shown in the bottom panels of Fig. 4. The UVB reveals hybridization between the Se $p_{z}$ and $\mathrm{W} d_{3 z^{2}-r^{2}}$ states at the $\Gamma$ point. Toward the $\mathrm{K}$ point, these contributions are gradually replaced by the Se $p_{x}+p_{y}$ and $\mathrm{W} d_{x^{2}-y^{2}}+d_{x y}$ states. By their out-of-plane orientations, the Se $p_{z}$ and $\mathrm{W} d_{3 z^{2}-r^{2}}$ states play no role for the spin splitting. The fact that the latter is determined by the Se $p_{x}+p_{y}$ and $\mathrm{W} d_{x^{2}-y^{2}}+d_{x y}$ states is confirmed by the similar behavior of $\Delta$ and the contributions of these orbitals as a function of $k_{\|}$. The aforementioned change of sign in $\Delta\left(k_{\|}\right)$reflects opposite effects of the Se $p_{x}+p_{y}$ and $\mathrm{W} d_{x^{2}-y^{2}}+d_{x y}$ states due to opposite directions of the potential gradient asymmetry on the Se and W sites; see Fig. 1(b). The competition is also visible in the spin-splitting behavior of the LCB. As the LCB at the K point is dominated by the $\mathrm{W} d_{3 z^{2}-r^{2}}$ states, $\Delta$ is much smaller than for the UVB.

From an applications point of view, the UVB is more promising than the LCB due to the enhanced spin splitting. Transport should rely on states close to the $\mathrm{K}$ point (maximal $\Delta$ ), while admixtures of states close to the $\Gamma$ point have to be avoided. Accordingly, the energy difference between the UVB at the $\Gamma$ point and at the $\mathrm{K}$ point $\left(\delta E_{\Gamma-\mathrm{K}}\right)$ should be as large as possible. From Fig. 1 one obtains $\delta E_{\Gamma-\mathrm{K}}=50$, 360, 220, and $530 \mathrm{meV}$ for $\mathrm{MoS}_{2}, \mathrm{MoSe}_{2}, \mathrm{WS}_{2}$, and $\mathrm{WSe}_{2}$, respectively. Thus, $\mathrm{WSe}_{2}$ is the most promising material. According to Figs. 3(c)-3(e), the character of the energy isosurface changes quite strongly with the binding energy, due to a finite value of $\delta E_{\Gamma-\mathrm{K}}$. For a binding energy of $0.8 \mathrm{eV}$, spin-splitted hole pocket pairs are found around the $\Gamma$ and $K / K^{\prime}$ points, pointing at a 
transport through mixed electronic states with large and small spin splittings. The $\mathrm{K} / \mathrm{K}^{\prime}$ pair shows a much larger separation between the two spin orientations than the $\Gamma$ pair, which is in accordance with an enhanced spin splitting at the $\mathrm{K} / \mathrm{K}^{\prime}$ point. For a binding energy of $0.5 \mathrm{eV}$, the $\Gamma$ pair vanishes since the energy at the $\Gamma$ point is below $E_{F}-\delta E_{\Gamma-\mathrm{K}}$. For a binding energy of $0.2 \mathrm{eV}$, only one spin species remains available near the $\mathrm{K} / \mathrm{K}^{\prime}$ point. Interestingly, the electronic states with binding energies below $\delta E_{\Gamma-\mathrm{K}}$ act as half-metallic states for $\vec{k}$ vectors directed to the corners of the first Brillouin zone.

\section{CONCLUSION}

In conclusion, the spin-orbit-induced spin splittings in monolayers of the transition-metal dichalcogenides $\mathrm{MoS}_{2}$, $\mathrm{MoSe}_{2}, \mathrm{WS}_{2}$, as well as $\mathrm{WSe}_{2}$ have been investigated by firstprinciples calculations, employing density functional theory. All four of our monolayer systems are identified as directband-gap semiconductors. Both the VBM and CBM appear at the $\mathrm{K}$ point of the Brillouin zone. It is found that spin-orbit coupling induces spin splittings of up to $456 \mathrm{meV}$ at the VBM due to a loss of the inversion symmetry of the bulk compounds in the monolayer case. Mainly the transition-metal $d_{x^{2}-y^{2}}+$ $d_{x y}$ states turn out to be responsible for the giant spin splitting. In addition, a full out-of-plane spin polarization results from the two-dimensional nature of both the electron motion and the potential gradient asymmetry. Since the Dyakanov-Perel spin relaxation is suppressed, very long spin lifetimes are characteristic for the studied systems. In particular, the $\mathrm{WSe}_{2}$ monolayer exhibits great potential for application, e.g., for the realization of the spin field-effect transistor. ${ }^{4,36}$ Controlling the spin-orbit-induced spin splitting requires a split gate instead of a top gate because of the in-plane nature of the potential gradient asymmetry. ${ }^{6}$ Remarkably, modifications of the electronic structure by the substrate can be avoided by preparing the monolayer systems on van der Waals-terminated surfaces due to an almost ideal electronic decoupling. ${ }^{29}$

\section{ACKNOWLEDGMENT}

We thank KAUST research computing for providing the computational resources used for this study. *udo.schwingenschlogl@kaust.edu.sa

${ }^{1}$ G. Bihlmayer, Y. M. Koroteev, P. M. Echenique, E. V. Chulkov, and

S. Blügel, Surf. Sci. 600, 3888 (2006).

${ }^{2}$ G. Dresselhaus, Phys. Rev. 100, 580 (1955).

${ }^{3}$ Y. A. Bychkov and E. I. Rashba, JETP Lett. 39, 78 (1984).

${ }^{4}$ S. Datta and B. Das, Appl. Phys. Lett. 56, 665 (1990).

${ }^{5}$ J. Schliemann, J. C. Egues, and D. Loss, Phys. Rev. Lett. 90, 146801 (2003).

${ }^{6}$ S. Bandyopadhyay and M. Cahay, Appl. Phys. Lett. 85, 1814 (2004).

${ }^{7}$ S. Bandyopadhyay and M. Cahay, Appl. Phys. Lett. 85, 1433 (2004).

${ }^{8}$ H. Tributsch, Z. Naturforsch. 32a, 972 (1977).

${ }^{9}$ Y. Kim, J. L. Huang, and C. M. Lieber, Appl. Phys. Lett. 59, 3404 (1991).

${ }^{10}$ J. Heising and M. G. Kanatzidis, J. Am. Chem. Soc. 121, 11720 (1999).

${ }^{11}$ X. Zong, H. Yan, G. Wu, G. Ma, F. Wen, L. Wang, and C. Li, J. Am. Chem. Soc. 130, 7076 (2008).

${ }^{12}$ K. F. Mak, C. Lee, J. Hone, J. Shan, and T. F. Heinz, Phys. Rev. Lett. 105, 136805 (2010).

${ }^{13}$ B. Radisavljevic, A. Radenovic, J. Brivio, V. Giacometti, and A. Kis, Nat. Nanotechnol. 6, 147 (2011).

${ }^{14}$ A. Kuc, N. Zibouche, and T. Heine, Phys. Rev. B 83, 245213 (2011).

${ }^{15}$ R. A. Bromley, R. B. Murray, and A. D. Yoffe, J. Phys. C 5, 759 (1972).

${ }^{16}$ T. Böker, R. Severin, A. Müller, C. Janowitz, R. Manzke, D. Voß, P. Krüger, A. Mazur, and J. Pollmann, Phys. Rev. B 64, 235305 (2001).

${ }^{17}$ C. Lee, H. Yan, L. E. Brus, T. F. Heinz, J. Hone, and S. Ryu, ACS Nano 4, 2695 (2010).

${ }^{18}$ J. N. Coleman, M. Lotya, A. ONeill, S. D. Bergin, P. J. King, U. Khan, K. Young, A. Gaucher, S. De, R. J. Smith, I. V. Shvets, S. K. Arora, G. Stanton, H.-Y. Kim, K. Lee, G. T. Kim, G. S. Duesberg, T. Hallam, J. J. Boland, J. J. Wang, J. F. Donegan, J. C. Grunlan, G. Moriarty, A. Shmeliov, R. J. Nicholls, J. M.
Perkins, E. M. Grieveson, K. Theuwissen, D. W. McComb, P. D. Nellist, and V. Nicolosi, Science 331, 568 (2011).

${ }^{19}$ P. Blaha, K. Schwarz, G. K. H. Madsen, D. Kvasnicka, and L. Luitz, WIEN2k, An augmented plane wave plus local orbitals program for calculating crystal properties (TU Vienna, Vienna, 2011).

${ }^{20}$ U. Schwingenschlögl and C. Schuster, Phys. Rev. Lett. 99, 237206 (2007); Europhys. Lett. 79, 27003 (2007); Eur. Phys. J. B 55, 43 (2007).

${ }^{21}$ U. Schwingenschlögl and C. Schuster, Chem. Phys. Lett. 439, 143 (2007); 435, 100 (2007).

${ }^{22}$ J. P. Perdew, K. Burke, and M. Ernzerhof, Phys. Rev. Lett. 77, 3865 (1996).

${ }^{23}$ Y. C. Cheng, Z. Y. Zhu, and U. Schwingenschlögl, Europhys. Lett. 95, 17005 (2011).

${ }^{24}$ Y. C. Cheng, Z. Y. Zhu, G. S. Huang, and U. Schwingenschlögl, Phys. Rev. B 83, 115449 (2011).

${ }^{25}$ R. Coehoorn, C. Haas, J. Dijkstra, C. J. F. Flipse, R. A. de Groot, and A. Wold, Phys. Rev. B 35, 6195 (1987).

${ }^{26}$ K. Kobayashi and J. Yamauchi, Phys. Rev. B 51, 17085 (1995).

${ }^{27}$ K. Fives, I. T. McGovern, R. McGrath, R. Cimino, G. Hughes, A. McKinley, and G. Thornton, J. Phys. Condens. Matter 4, 5639 (1992).

${ }^{28}$ R. Coehoorn, C. Haas, and R. A. de Groot, Phys. Rev. B 35, 6203 (1987).

${ }^{29}$ A. Klein, S. Tiefenbacher, V. Eyert, C. Pettenkofer, and W. Jaegermann, Phys. Rev. B 64, 205416 (2001).

${ }^{30}$ M. Traving, M. Boehme, L. Kipp, M. Skibowski, F. Starrost, E. E. Krasovskii, A. Perlov, and W. Schattke, Phys. Rev. B 55, 10392 (1997).

${ }^{31}$ S. LaShell, B. A. McDougall, and E. Jensen, Phys. Rev. Lett. 77, 3419 (1996).

${ }^{32}$ Y. M. Koroteev, G. Bihlmayer, J. E. Gayone, E. V. Chulkov, S. Blügel, P. M. Echenique, and P. Hofmann, Phys. Rev. Lett. 93, 046403 (2004). 
${ }^{33}$ J. Henk, A. Ernst, and P. Bruno, Phys. Rev. B 68, 165416 (2003).

${ }^{34}$ Y. Ohno, R. Terauchi, T. Adachi, F. Matsukura, and H. Ohno, Phys. Rev. Lett. 83, 4196 (1999).

${ }^{35}$ Y. S. Gui, C. R. Becker, N. Dai, J. Liu, Z. J. Qiu, E. G. Novik, M. Schäfer, X. Z. Shu, J. H. Chu, H. Buhmann, and L. W. Molenkamp, Phys. Rev. B 70, 115328 (2004).

${ }^{36}$ J. Nitta, T. Akazaki, H. Takayanagi, and T. Enoki, Phys. Rev. Lett. 78, 1335 (1997).
${ }^{37}$ K. Yaji, Y. Ohtsubo, S. Hatta, H. Okuyama, K. Miyamoto, T. Okuda, A. Kimura, H. Namatame, M. Taniguchi, and T. Aruga, Nat. Commun. 1, 1 (2009).

${ }^{38}$ P. D. C. King, R. C. Hatch, M. Bianchi, R. Ovsyannikov, C. Lupulescu, D. Guan, J. L. Mi, E. D. L. Rienks, J. Fink, A. Lindblad, S. Svensson, S. Bao, B. B. Iversen, W. Eberhardt, F. Baumberger, and Ph. Hofmann, Phys. Rev. Lett. 107, 096802 (2011).

${ }^{39}$ M. Hochstrasser, J. G. Tobin, E. Rotenberg, and S. D. Kevan, Phys. Rev. Lett. 89, 216802 (2002). 